\title{
The impact of increasing education levels on rising life expectancy: a decomposition analysis for Italy, Denmark, and the USA
}

\author{
Marc Luy ${ }^{1,2^{*}}$, Marina Zannella ${ }^{1,2,7}$, Christian Wegner-Siegmundt ${ }^{1,2}$, Yuka Minagawa ${ }^{5}$, Wolfgang Lutz ${ }^{1,2,3,4}$
} and Graziella Caselli ${ }^{6}$

\author{
* Correspondence: \\ marc.luy@oeaw.ac.at \\ ${ }^{1}$ Wittgenstein Centre for \\ Demography and Global Human \\ Capital (IIASA, VID/ÖAW, WU), \\ Welthandelsplatz 2 / Level 2, 1020 \\ Vienna, Austria \\ ${ }^{2}$ Vienna Institute of Demography of \\ the Austrian Academy of Sciences, \\ Welthandelsplatz 2 / Level 2, 1020 \\ Vienna, Austria \\ Full list of author information is \\ available at the end of the article
}

\begin{abstract}
Significant reductions in mortality are reflected in strong increases in life expectancy particularly in industrialized countries. Previous analyses relate these improvements primarily to medical innovations and advances in health-related behaviors. Mostly ignored, however, is the question to what extent the gains in life expectancy are related to structural changes in the populations due to increasing education levels. We decompose changes of the total populations' life expectancy at age 30 in Italy, Denmark, and the USA, over the 20-year period between 1990 and 2010 into the effects of education-specific mortality changes ("M effect") and changes in the populations' educational structure ("P effect"). We use the "replacement decomposition technique" to further subdivide the $\mathrm{M}$ effect into the contributions by the individual education groups. While most of the increases in life expectancy are due to the effect of changing mortality, a large proportion of improvements in longevity can indeed be attributed to the changing structure of the population by level of education in all three countries. The estimated contribution of the $P$ effect ranges from around $15 \%$ for men in the USA to approximately $40 \%$ for women in Denmark. This study demonstrates strong associations between education and overall population health, suggesting that education policies can also be seen as indirect health policies.
\end{abstract}

Keywords: Life expectancy, Human capital, Education, Population structure, Decomposition; Health policy

\section{Introduction}

Reductions in mortality which started in Europe in the middle of the eighteenth century have continued to spread globally and led to huge increases in life expectancy, with industrialized countries being most advanced in this process. Most of these changes were due to a shift in cause-specific mortality patterns from communicable diseases at younger ages, including neo-natal and childhood ages, to non-communicable conditions more prevalent at advanced ages, as described in Omran's (1971) "epidemiologic transition theory." Recently, the potentials of human longevity were further extended by the "cardiovascular revolution" which started in the 1970s and initiated a new period of decreasing mortality (Caselli 2015; Olshansky and Ault 1986; Omran 1983; Rogers and Hackenberg 1987). Studies have tended to relate these improvements to public health measures and new medical advancements, such

(c) The Author(s). 2019 Open Access This article is distributed under the terms of the Creative Commons Attribution 4.0 International License (http://creativecommons.org/licenses/by/4.0/), which permits unrestricted use, distribution, and reproduction in any medium, provided you give appropriate credit to the original author(s) and the source, provide a link to the Creative Commons license, and indicate if changes were made. 
as developments in screening, prevention, and treatment of cardiovascular diseases (Mackenbach et al. 1988; Vallin and Meslé 2004; Wilmoth 2000). Improvements in living conditions and health-related behaviors have also reduced mortality, thereby leading to further increase in survival chances (Denney et al. 2010; Janssen and Kunst 2005; Rogers et al. 1999, 2010).

In tandem with the decrease of mortality, the populations have experienced significant increases in educational attainment (Lutz et al. 2014a). Between 1970 and 2009, for instance, the average number of years of education rose by 3.6 years for people aged 25 and older in 175 countries (Gakidou et al. 2010). A great deal of research acknowledges the strong impact of socioeconomic resources on various health outcomes, suggesting socioeconomic conditions as a "fundamental cause" of inequalities in health and mortality (Link and Phelan 1995). Many studies have shown that highly educated people stand to benefit first and most from the prevailing drivers of decreasing mortality, particularly with regard to the risk factors of cardiovascular diseases (Hummer and Lariscy 2011; Mirowsky and Ross 2003). These inequalities translate into differences in life expectancy of several years between individuals with low and high educational attainment (Caselli et al. 2014). Lleras-Muney (2005) estimated-with data for the USA in 1960-that one more year of education increased average life expectancy at age 35 by as much as 1.7 years. Further, Woolf et al. (2007) estimated that eliminating education-specific inequalities in mortality would save even more lives than medical advances.

Naturally, higher education does not automatically lead to better health. It is also unclear to what extent education itself plays a direct role inside the complex network of various health-related socioeconomic factors (Kaplan et al. 2014). Although evidence infers that it is justified to assume some direct functional causality (Geyer and Peter 2000; Lleras-Muney 2005; Lutz and Skirbekk 2014; van Kippersluis et al. 2011), it appears that the association between education and mortality operates to a larger extent along an indirect pathway in which education acts as access criterion to other drivers of health and longevity (Arendt 2005; Clark and Royer 2013; Davey Smith et al. 1998; Kilpi et al. 2016; Kröger et al. 2015; Lager and Torssander 2012). As Hayward et al. (2015: 16) put it, "...there is no inherent causal association between educational attainment and adult mortality; instead, the causal association is dependent upon time, place, and social environment under study."

Here, we briefly summarize the major mechanisms linking education to health and mortality (an extensive compilation of recent studies can be found in Montez and Friedman (2015)). Schooling develops basic cognitive functioning, such as reading, writing, and communicating, and teaches individuals how to think logically, critically analyze data, solve problems, and implement plans (Kingston et al. 2003). Higher education is the key to stable and well-paid jobs, and increased income helps to pay for nutritious food, better-quality housing, and high-quality medical care (Mirowsky and Ross 2003). In addition, education promotes healthy lifestyles through the development of effective human agency. Highly educated people use their knowledge, information, and past experiences to avoid health-related risk factors and engage in health-enhancing behaviors, such as smoking cessation, alcohol abstinence, and frequent physical exercise (Denney et al. 2010; Laaksonen et al. 2008). Moreover, education provides socio-psychological resources that can contribute to health and longevity through emotional and instrumental support (House et al. 1988). That well-educated people are 
more likely to be and remain married also contributes to the relationship between education and health (Qian and Preston 1993).

These examples indicate how higher education contributes to better health and longer lives on the individual level. Furthermore, investments in education also improve the health status on the population level. Baker et al.'s (2011) systematic review demonstrates the strong relationship between education and adult mortality across countries at different levels of development. These insights raise the question to what extent the increasing education levels constitute an additional factor behind populations' rising life expectancy. If educational attainment improves in a population, it usually follows that the proportion of individuals with lower education levels decreases, while the proportion of those with higher education increases. Consequently, the health of the overall population should increase accordingly, even if the mortality rates of each education category remain unchanged. The question to what extent the gains in life expectancy were caused by such changes in the populations' education structure, in addition to the effect of decreasing mortality within the education groups, has been addressed only rarely so far. Shkolnikov et al. (2006) and Jasilionis et al. (2007) provided such estimates for some northern, central, and eastern European countries between the late 1980s and 1990s. They found that the changes in the education-specific composition had in fact a remarkable positive effect on the populations' life expectancy levels. Similar conclusions were derived in studies on specific national subpopulations (De Grande et al. 2014; Klein et al. 2006; Turra et al. 2016).

Guided by the works of Shkolnikov et al. (2006) and Jasilionis et al. (2007), we examine, on the basis of the same methodology, how changes in the educational attainment by age and sex contributed to increasing overall life expectancy at age $30\left(e_{30}\right)$ in Italy, Denmark, and the USA between around 1990 and 2010. Thus, our study extends the literature on the relationship between populations' levels of education and life expectancy by adding insights from new populations and by analyzing a longer and more recent observation period. Also, the basic research question differs from the previous studies. Shkolnikov et al. (2006) and Jasilionis et al. (2007) examined primarily whether the stalls and decreases of life expectancy in central and eastern Europe during the 1980s were driven by certain education-specific subpopulations. The present study focuses, by contrast, on western populations and the specific question to what extent changes in the educational structure of the populations contributed to their steady increases in life expectancy.

The populations of Italy, Denmark, and the USA form an interesting sample to assess the relationship between populations' levels of education and life expectancy because they differ in terms of life expectancy, magnitudes of education-specific differentials in mortality, and the education profile of the populations. In the USA, formal schooling lasts 13 years until around age 18 with some variations between the states. Notably, the US population experienced substantial changes over the years in terms of the levels, content, and importance of education (Hummer and Lariscy 2011). The proportion of the population at age 25 and older with at least high school education more than doubled since the early 1960s to almost 90\% in 2015 (Ryan and Bauman 2016). In the two European states, compulsory education is shorter than in the USA (OECD 2016). Also, the process of education expansion started later. The proportion of adults with tertiary education is therefore still distinctly lower, above all in Italy (OECD 2016). 
More effective population policies in the past let Denmark move ahead in the process of education expansion (Arendt 2005), giving the country the intermediate position among the three study populations. All these characteristics of the national education systems refer exclusively to quantitative aspects. Certainly, education systems differ also in qualitative aspects. These are, however, not the topic of this study. We will come back to this issue briefly in the "Discussion" section of the paper.

In the following two sections, we describe the data and methods we used to construct the life tables by education and to decompose the changes in $e_{30}$ over the observed 20-year period into the effects of changing education-specific mortality ("M effect") and changing educational structure of the populations ("P effect"). In the "Results" section, we summarize the outcomes of our analyses which confirm that increasing education levels are strongly related to the rising levels of life expectancy. This finding has policy implications, particularly for newly industrializing countries and other populations of the global south where education levels are projected to increase even more rapidly than in the industrialized world (Lutz et al. 2014a). Because further rises of average education levels are bound to occur by the fact that in most countries the younger cohorts are better educated than the older ones, our results also fuel the expectation of continuing increases in life expectancy-an issue that is highly controversial among demographers and split the community into "optimists" and "pessimists". All these issues and the limitations of the study are discussed in the final section of the paper.

\section{Data}

The analysis requires age- and education-specific data for the populations at risk and the deaths for the start and end years of our observation period. Our study starts at age 30 because this is the age when education is usually completed. We defined educational attainment on the basis of the ISCED-97 scale (UNESCO 1996) with the three levels low (ISCED 0-2), medium (ISCED 3-4), and high education (ISCED 5-6). Obtaining this data for our study populations was a challenging task, in particular for the early 1990s. Because the aim of this study is to estimate the impact of changes in populations' educational structures on overall life expectancy, we gave priority to the most similar derivation of life tables for the studied populations rather than the most accurate estimate of life expectancy by education for each population. A cross-country comparison of differentials in life expectancy by education level would be a different research question which is not only affected by the characteristics of the data sources. The same formal education levels can include significant differences in the quality of education. Finally, the number of considered education levels plays a role with regard to both, the extent of differentials between the levels and the estimation of the effects of corresponding structural changes. The following paragraphs summarize the data sources and how we constructed our database as homogeneous as possible.

Italy

Information about the population by age, sex, and education level was taken from Italian census data which is available online at the data warehouse of the National Statistical Institute of Italy (ISTAT). ${ }^{1}$ Age-, sex-, and education-specific numbers of death stem from the Italian national vital statistics registry on causes of death and are based 
on death certificates. Data for 2011 were downloaded from the ISTAT data warehouse. Data for 1991 are not available online and were manually collected and digitalized by the authors from the printed reports "Nascite e decessi" at the ISTAT archive in Rome, Italy. In all data sources, education refers to the highest educational attainment which allowed us to translate the numbers directly into the ISCED-97 classification. The data for the population at risk and the number of deaths cover the ages 30 to $85+$ in 5-year age groups. Cases with unknown education level (4.3\% of deaths in 1991) have been distributed according to the education-specific proportions for valid cases for each age and sex group. Because of the introduction of new data-check plans in 2004, there were no cases with missing information in the data for 2011.

\section{Denmark}

Information about the population by age, sex, and education level comes from nationwide population registers, covering the ages 30 to 100. Information about age-, sex-, and education-specific mortality was available in form of probabilities of dying for ages 30 to 64 . These were derived by register linkage at the individual level by use of the unique personal identification number assigned to all Danish citizens (Thygesen et al. 2011). For ages 65 and older, age- and sex-specific probabilities of dying were only available for the total population without separation by education level. All data were available for single ages and represent averages of two subsequent calendar years (i.e., the data for 1990 refers to the average of 1989 and 1990, and the data for 2010 to the average of 2009 and 2010). The databases did not include any cases with missing information. Education level was defined according to ISCED-97 and categorized into the levels 1-2, 3A, 3C \& 4A, and 5-6. We merged the data for ISCED levels 3A, 3C, and 4A (ISCED 3-4) and derived the age-, sex-, and education-specific numbers of death from the data for the population and the corresponding probabilities of dying. Education-specific probabilities of dying above age 64 were derived by extrapolating the available data for ages 30 to 64 with the Brass logit life table model (Brass 1971). We used the age-specific mortality schedules of the total Danish population by sex (for 1989 respective to 2009) from the Human Mortality Database (2017) as standard life tables. ${ }^{2}$ The Brass parameters were estimated from the age- and education-specific probabilities of dying at ages 30 to 64 (see the "Life tables by education level" section for more details on our estimations with the Brass model).

\section{USA}

Information about the population by age, sex, and education level stems from the data collection IPUMS-USA (Ruggles et al. 2015). ${ }^{3}$ Age-, sex-, and education-specific numbers of death stem from the Division of Vital Statistics of the National Center for Health Statistics and are based on death certificates. The data were downloaded from the website of the Centers for Disease Control and Prevention (CDC). ${ }^{4}$ Both datasets are available in micro-data form, and we merged them into 5-year age groups from age 30 to $90+$. In the data for the population at risk, education refers to the highest educational attainment, which enabled us to translate the numbers directly into the ISCED-97 classification. In the mortality data for 1990, education is classified by the number of actual years of schooling. In the mortality data for 2010, education refers to 
the highest educational attainment as defined in the Revision of the Standard Certificate of Death of the year $2003 .^{5}$ Given that not all states have adopted the new classification, the mortality data for 2010 includes both classifications: number of years in school and the highest educational attainment. For the states which used the new regulation, we were able to translate the numbers directly into the ISCED-97 classification. To obtain corresponding education classifications for the deaths of 1990 and for those of 2010 which were still classified by the number of school years, we performed an approximate reclassification into the ISCED-97 system on the basis of the EDATTAIN International Recode of Educational Attainment. ${ }^{6}$ Cases with unknown education levels (23.6\% of deaths in 1990 and $2.2 \%$ of deaths in 2010) were distributed according to the education-specific proportions for valid cases for each age and sex group.

\section{Methods}

As described in the previous section, the characteristics of the underlying data differ between our study populations, e.g., with regard to the construction of the three ISCED education groups. Another important difference concerns the data sources for the education-specific populations at risk and the corresponding number of deaths. Whereas for Denmark both stem from linked population registers, we have different and unlinked data sources for Italy and the USA. Consequently, the estimated education-specific differentials in life expectancy cannot be directly compared between the populations. However, as outlined at the beginning of the "Data" section and later in more detail in the "Discussion" section of this paper, the purpose of this study lies exclusively in the estimation of the relationship between increasing education levels and overall life expectancy. In order to compare results across our study populations, we constructed life tables as basis for the decomposition analysis. Life tables were constructed as consistently as possible in regards to the available data for each study population.

\section{Life tables by education level}

We constructed abridged period life tables from age 30 for 5-year age groups for the populations of Italy (1991 and 2011), Denmark (1990 and 2010), and the USA (1990 and 2010) on the basis of age-, sex-, and education-specific death rates derived from the data described in the "Data" section. The age-specific fractions of last age interval of life were estimated with the Keyfitz method (Preston et al. 2001). In order to get a homogeneous basis for our analysis, we extrapolated all life tables with the Brass logit model (Brass 1971) to age 110. The Brass parameters were estimated from the age- and education-specific probabilities of dying at ages 30 to 64 (because of the restriction set by the data for Denmark, see the "Denmark" section). Country- and period-specific life tables from the Human Mortality Database (2017) were used as standard. ${ }^{7}$ The extrapolated life tables for each sex- and education-specific subpopulation were then used to derive the death rates for the country-specific last open age intervals. These start at different ages as determined by the country-specific availability of data for the living population (see the "Data" section). The basic data on deaths and populations at risk by education levels and the life tables by educational attainment for each country and observation period are available in the Additional file 1. 


\section{Decomposition of changes in life expectancy}

The central aim of this study is to decompose the changes in life expectancy at age 30 $\left(e_{30}\right)$ for the total populations of Italy, Denmark, and the USA between around 1990 (time $t 1$ ) and around 2010 (time $t 2$ ) into the contributions of changes in education-specific mortality ( $M$ effect) and changes in the populations' educational structure (P effect). The terms are adopted from Shkolnikov et al. (2006) who introduced this specific decomposition approach. Isolating the $\mathrm{M}$ and $\mathrm{P}$ effects is difficult because they are complexly interrelated. On the one hand, changes in education-specific mortality cause changes in the education-specific structure of the population through increasing or decreasing survival chances. On the other hand, changes in the educational structure of the population affect the corresponding mortality levels because the risk compositions of the education-specific subpopulations are changing, e.g., when a larger proportion of formerly less-educated individuals with higher mortality risks move to higher education levels or vice versa. Between $t 1$ and $t 2$, both changes occur simultaneously with different and varying magnitudes what makes the interrelation even more complex.

Although several approaches have been proposed to decompose the change in the death rates, they all follow the same basic idea of standardization, that is to isolate the effect of changing mortality under constant population composition, or vice versa, the effect of changing composition of the population under constant mortality. The challenging issue is to decide the condition for which mortality and population compositions are standardized for. Three different standards are possible: (1) the conditions prevailing at $t 1,(2)$ the conditions prevailing at $t 2$, and (3) the average of conditions prevailing at $t 1$ and $t 2$. We used the replacement decomposition technique (Andreev et al. 2002; Shkolnikov et al. 2003) which estimates the M and P effects on the basis of standard (3). The basic approach of this method is to apply a "[...] stepwise replacement of elements from one vector of age-specific mortality rates by respective elements of another vector" (Andreev et al. 2002: 500).

In a nutshell, the replacement decomposition technique works as follows. Starting with the original vector of education-specific death rates at $t 1$, we estimate $e_{30}$. In the first replacement step, the education-specific death rates of the age group 30-34 of $t 1$ are substituted by the corresponding death rates of $t 2$, while the educational structure of the population is kept constant at the level of $t 1$. The difference between the resulting modeled values for $e_{30}$ at $t 2$ and those of the start year $t 1$ provides an estimate for the $\mathrm{M}$ effect of the age group 30-34 on the basis of standard (1). In the second step, the P effect originating from education-specific structural changes in this age group is estimated accordingly by substituting the population's educational structure in age group 30-34 of $t 1$ by those of $t 2$, while the education-specific death rates are kept constant at the level of $t 1$. These steps are repeated for all possible combinations of age- and education-specific death rates and the population's educational structure until the conditions prevailing at $t 1$ are completely replaced by the corresponding conditions at $t 2$. Then, the procedure is performed equivalently on the basis of standardization procedure (2) for the transition from $t 2$ to $t 1$ through substituting the education- and age-specific death rates and the educational structure at $t 2$ stepwise by those prevalent at $t 1$.

The different directions across the replacement paths, the subpopulations, and the age schedule cause different combinations of vectors which should be fully considered 
in an ideal decomposition procedure. To avoid the corresponding bulk of calculations, the usually applied strategy is to perform the replacement procedure only from the youngest to the oldest age group. The resulting set of age-specific $\mathrm{M}$ and $\mathrm{P}$ effects is then summed up to the overall effects and averaged for the results obtained by standards (1) and (2). Consequently, the yielded estimates can be regarded as approximate overall $\mathrm{M}$ and $\mathrm{P}$ effects, i.e., estimates for the contributions of changes in education-specific mortality (respective contributions of changes in the educational structure of the population) to changing death rates between $t 1$ and $t 2$, when the population's educational structure (respective mortality conditions) remain constant. The particular advantage of the replacement decomposition technique is that the replacement can be performed separately for each education subgroup. This allows a subdivision of the $\mathrm{M}$ effect into the individual contributions of education group-specific changes in mortality (more and specific details about the method can be found in Shkolnikov et al. 2006). For our analyses, we used the MS Excel macro provided by Andreev and Shkolnikov (2012). ${ }^{8}$

\section{Results}

Table 1 presents the distribution of and changes in educational attainment at ages 30 and above for Italy, Denmark, and the USA during the study period. Substantial changes have been made in educational attainment levels in these countries between 1990 and 2010. The proportion of the population with low education strongly declined, and consequently, there were large increases of the proportions in the medium and high education categories. Italy had massive shifts particularly between the low and medium levels, while the proportion of highly educated people increased somewhat less. Denmark experienced similar decreases at the low education level but with more distinct differences between women and men. In contrast to Italy, the high education

Table 1 Proportion (in percent) of populations aged 30 years and above by education level for Italy (1991-2011), Denmark (1990-2010), and the USA (1990-2010), stratified by gender

\begin{tabular}{|c|c|c|c|c|c|c|c|c|c|}
\hline & \multicolumn{3}{|c|}{ Italy (1991-2011) } & \multicolumn{3}{|c|}{ Denmark (1990-2010) } & \multicolumn{3}{|c|}{ USA (1990-2010) } \\
\hline & Low & Med & High & Low & Med & High & Low & Med & High \\
\hline \multicolumn{10}{|l|}{$\overline{M e n}$} \\
\hline 1990/91 & 75.9 & 17.6 & 6.5 & 48.6 & 35.0 & 16.4 & 21.7 & 54.7 & 23.6 \\
\hline 2010/11 & 53.8 & 34.0 & 12.2 & 29.6 & 43.9 & 26.5 & 13.3 & 57.9 & 28.8 \\
\hline Difference & -22.1 & +16.4 & +5.7 & -19.0 & +8.9 & +10.1 & -8.4 & +3.2 & +5.2 \\
\hline \multicolumn{10}{|l|}{ Women } \\
\hline 1990/91 & 81.8 & 14.3 & 3.9 & 62.7 & 23.4 & 13.9 & 22.9 & 60.1 & 17.0 \\
\hline 2010/11 & 56.8 & 30.7 & 12.5 & 35.0 & 36.3 & 28.7 & 12.4 & 60.4 & 27.2 \\
\hline Difference & -25.0 & +16.4 & +8.6 & -27.7 & +12.9 & +14.8 & -10.5 & +0.3 & +10.2 \\
\hline \multicolumn{10}{|l|}{ Both sexes } \\
\hline 1990/91 & 79.0 & 15.8 & 5.2 & 55.9 & 29.0 & 15.1 & 22.4 & 57.6 & 20.0 \\
\hline 2010/11 & 55.4 & 32.2 & 12.4 & 32.3 & 40.1 & 27.6 & 12.8 & 59.2 & 28.0 \\
\hline Difference & -23.6 & +16.4 & +7.2 & -23.6 & +11.1 & +12.5 & -9.6 & +1.6 & +8.0 \\
\hline
\end{tabular}

Source: authors' own calculations; the data sources for the three populations can be found in the "Data" section Notes: Low education includes the International Standard Classification of Education (ISCED-97) levels 0 (pre-primary education), 1 (primary education or first stage of basic education), and 2 (lower secondary education); medium education includes the levels of 3 (upper secondary education) and 4 (post-secondary non-tertiary education); high education refers to the levels of 5 (first stage of tertiary education) and 6 (second stage of tertiary education). Differences are given in percentage points 
category increased more than the medium category in both sexes. Partly due to starting from a higher level, the USA saw considerably smaller increases in the education levels over the same period. Nonetheless, the share of those with low educational attainment also declined during the study period, while the proportion of medium education level changed only slightly. Hence, the shift in education level occurred primarily at the extreme ends of low and high education levels. Despite these increases in education levels in all three countries, large proportions of the populations still remained in the low education groups, mostly concentrated in the older ages. In the most recent observation years, $55.4 \%$ of the Italian population had less than upper secondary education, and the respective figures are $32.3 \%$ in Denmark and $12.8 \%$ in the USA.

Table 2 summarizes how life expectancy at age $30\left(e_{30}\right)$ increased during the 20 years of observation and how it varied by education group in the three countries. For the total populations, $e_{30}$ increased by 4.4 years in Italy, 3.6 years in Denmark, and 2.8 years in the USA. In all populations, there was a clear gradient in $e_{30}$ by level of education. Those with high education had the highest life expectancy, followed by medium and low education groups (except for Italian women in 1991 and the total Italian population in 2011). Two points are worth emphasizing. First, the pattern of impact of education on life expectancy was different among countries. In Italy, the largest gap in $e_{30}$ existed between the low education level and the two others, suggesting that completion of upper secondary education was a mortality threshold in the Italian population during the 1990s and 2000s. By contrast, tertiary education seems to have been the key to life expectancy improvements in the USA: differences between medium and high education were larger than those between medium and low education. Denmark shows the smallest inequalities across education groups, albeit with more notable sex differences in the highest education category. Second, educational differences in $e_{30}$ have widened over time in most cases. The differentials between high and low education groups were consistently larger in more recent years for both sexes in all three countries. Increases can be observed between medium and low education as well, though the extent of

Table 2 Life expectancy at age 30 (e30) for Italy (1991-2011), Denmark (1990-2010), and the USA (1990-2010), stratified by education and gender

\begin{tabular}{|c|c|c|c|c|c|c|c|c|c|c|c|c|}
\hline & \multicolumn{4}{|c|}{ Italy (1991-2011) } & \multicolumn{4}{|c|}{ Denmark (1990-2010) } & \multicolumn{4}{|c|}{ USA (1990-2010) } \\
\hline & $\overline{\text { Total }}$ & Low & Med & $\overline{\text { High }}$ & Total & Low & Med & $\overline{\text { High }}$ & Total & Low & Med & High \\
\hline \multicolumn{13}{|l|}{ Men } \\
\hline 1990/91 & 45.5 & 44.7 & 49.1 & 51.4 & 43.4 & 42.2 & 43.5 & 46.3 & 44.2 & 41.7 & 43.7 & 47.1 \\
\hline 2010/11 & 50.6 & 48.3 & 56.1 & 56.3 & 47.6 & 44.6 & 47.8 & 50.8 & 48.0 & 43.2 & 47.2 & 52.6 \\
\hline Difference & +5.1 & +3.6 & +7.0 & +4.9 & +4.2 & +2.4 & +4.3 & +4.5 & +3.8 & +1.5 & +3.5 & +5.5 \\
\hline \multicolumn{13}{|l|}{ Women } \\
\hline 1990/91 & 51.5 & 51.2 & 56.1 & 55.4 & 48.6 & 47.9 & 47.7 & 51.6 & 50.3 & 49.5 & 49.8 & 51.5 \\
\hline 2010/11 & 55.1 & 53.8 & 60.4 & 60.5 & 51.5 & 49.1 & 52.8 & 54.4 & 52.2 & 48.8 & 51.9 & 55.3 \\
\hline Difference & +3.6 & +2.6 & +4.3 & +5.1 & +2.9 & +1.2 & +5.1 & +2.8 & +1.9 & -0.7 & +2.1 & +3.8 \\
\hline \multicolumn{13}{|l|}{ Both sexes } \\
\hline 1990/91 & 48.6 & 48.1 & 52.3 & 52.6 & 46.0 & 45.2 & 45.3 & 48.7 & 47.4 & 45.5 & 47.0 & 49.0 \\
\hline 2010/11 & 53.0 & 51.2 & 58.1 & 57.9 & 49.6 & 47.0 & 49.7 & 52.8 & 50.2 & 45.8 & 49.7 & 53.8 \\
\hline Difference & +4.4 & +3.1 & +5.8 & +5.3 & +3.6 & +1.8 & +4.4 & +4.1 & +2.8 & +0.3 & +2.7 & +4.8 \\
\hline
\end{tabular}

Source: authors' own calculations; the data sources for the three populations can be found in the "Data" section Note: Decimals may be adjusted to make differences fit to results of decomposition in Table 3 
changes compared to those between low and high education differed between populations and sexes. The only exceptions are the decreasing differences between high and medium education among men and the total population in Italy, and among women in Denmark.

Results from the decomposition analyses are summarized in Table 3 and in Figs. 1 and 2. It becomes apparent that improvements in $e_{30}$ were driven primarily by the $\mathrm{M}$ effect caused by decreasing education-specific death rates. The $M$ effect was responsible for $80.9 \%$ of the total gain in $e_{30}$ in the USA, for $80.1 \%$ in Italy, and for $75.6 \%$ in Denmark. As expected, the pattern of the $\mathrm{M}$ effect broken down into specific education categories was not uniform across countries. In Italy, most of the $\mathrm{M}$ effect was caused by the low education group, resulting in a $61.5 \%$ increase in $e_{30}$ of the total population between 1991 and 2011 (57.9\% for men; 67.7\% for women). In the USA, the mortality reductions in the medium education category made the largest contribution to the increase in $e_{30}$ (51.5\% for the total population; $48.4 \%$ for men; $62.5 \%$ for women). The low education group contributed least to increasing life expectancy in the USA. Among women, the contribution was even negative due to the decrease of $e_{30}$ between 1990 and 2010 (see Table 2). In Denmark, no specific education group dominated the mortality decline. The highest contribution originated from the low education level

Table 3 Contributions of changes in mortality ( $M$ effect) and populations' educational structure ( $P$ effect) to differences in life expectancy at age 30 (e $\left.e_{30}\right)$ for Italy (1991-2011), Denmark (1990-2010), and the USA (1990-2010), stratified by gender

\begin{tabular}{|c|c|c|c|c|c|c|}
\hline & \multicolumn{2}{|l|}{ Italy } & \multicolumn{2}{|c|}{ Denmark } & \multicolumn{2}{|l|}{ USA } \\
\hline & Years & Percent & Years & $\overline{\text { Percent }}$ & Years & Percent \\
\hline \multicolumn{7}{|l|}{ Men } \\
\hline Total difference in $e_{30}$ & 5.1 & 100.0 & 4.2 & 100.0 & 3.8 & 100.0 \\
\hline M effect (mortality) & 4.0 & 78.8 & 3.2 & 78.1 & 3.2 & 83.6 \\
\hline Low education & 2.9 & 57.9 & 1.4 & 34.6 & 0.3 & 8.6 \\
\hline Medium education & 0.8 & 15.9 & 1.2 & 28.3 & 1.9 & 48.4 \\
\hline High education & 0.3 & 5.0 & 0.6 & 15.2 & 1.0 & 26.6 \\
\hline P effect (structure) & 1.1 & 21.2 & 1.0 & 21.9 & 0.6 & 16.4 \\
\hline \multicolumn{7}{|l|}{ Women } \\
\hline Total difference in $e_{30}$ & 3.6 & 100.0 & 2.9 & 100.0 & 1.9 & 100.0 \\
\hline M effect (mortality) & 2.9 & 80.4 & 1.8 & 60.5 & 1.5 & 79.0 \\
\hline Low education & 2.4 & 67.7 & 0.8 & 26.4 & -0.2 & -10.0 \\
\hline Medium education & 0.3 & 8.9 & 0.7 & 23.1 & 1.2 & 62.5 \\
\hline High education & 0.2 & 3.8 & 0.3 & 11.0 & 0.5 & 26.5 \\
\hline P effect (structure) & 0.7 & 19.6 & 1.1 & 39.5 & 0.4 & 21.0 \\
\hline \multicolumn{7}{|l|}{ Both sexes } \\
\hline Total difference in $e_{30}$ & 4.4 & 100.0 & 3.6 & 100.0 & 2.8 & 100.0 \\
\hline M effect (mortality) & 3.5 & 80.1 & 2.7 & 75.6 & 2.3 & 80.9 \\
\hline Low education & 2.7 & 61.5 & 1.2 & 33.4 & 0.0 & 1.3 \\
\hline Medium education & 0.6 & 13.5 & 1.0 & 27.1 & 1.5 & 51.5 \\
\hline High education & 0.2 & 5.1 & 0.5 & 15.1 & 0.8 & 28.1 \\
\hline P effect (structure) & 0.9 & 19.9 & 0.9 & 24.4 & 0.5 & 19.1 \\
\hline
\end{tabular}

Source: authors' own calculations; the data sources for the three populations can be found in the "Data" section Note: Results in percent are derived from exact changes (in years) and may not correspond to percentages of rounded values; decimals may be adjusted to add up to total values 


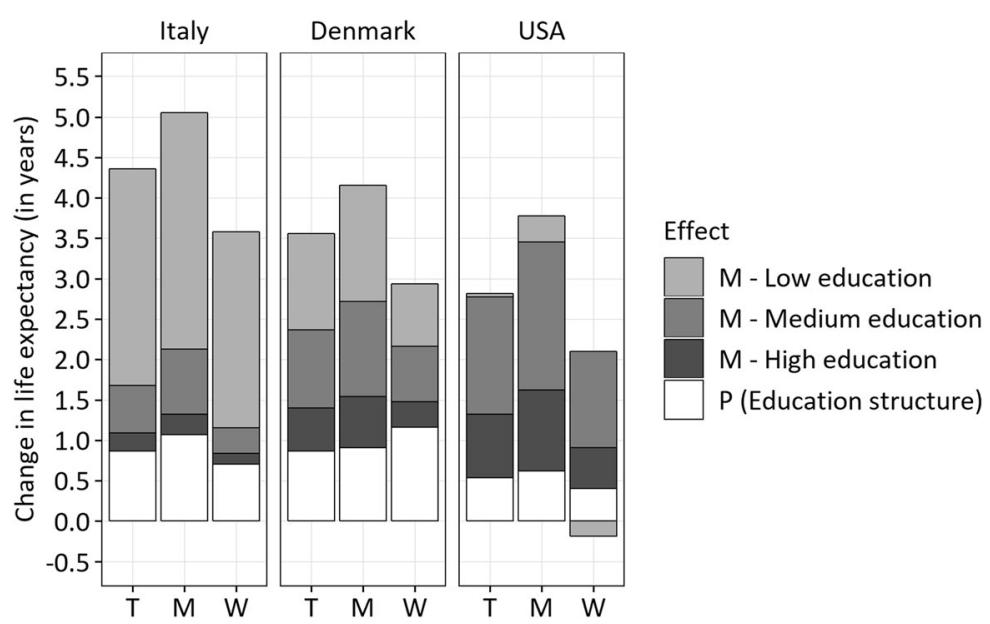

Fig. 1 Absolute contributions of changes in mortality ( $M$ effect) and populations' educational structure (P effect) to differences in life expectancy at age 30 (in years) for Italy (1991-2011), Denmark (1990-2010), and the USA (19902010), stratified by gender. Source: authors' own calculations; the data sources for the three populations can be found in the "Data" section. Note: T refers to total population, $\mathrm{M}$ is men, and W is women

(33.4\% for the total population; 34.6\% for men; $26.4 \%$ for women), but the contribution of the medium education level was not much smaller (27.1\% for the total population; $28.3 \%$ for men; $23.1 \%$ for women). The contribution of mortality declines in the high education level ranged from $3.8 \%$ among Italian women to $28.1 \%$ in the total population of the USA.

While the most powerful contributor to increasing life expectancy was the effect of decreasing mortality within education groups, the changing educational structure of the populations also contributed substantially to the increase in $e_{30}$ in all three countries (see Fig. 1). In Italy and Denmark, the structural change in the populations accounted for approximately 1 year of the increases in $e_{30}$ between around 1990 and 2010, and in the USA for about half a year. The smaller absolute increase attributable

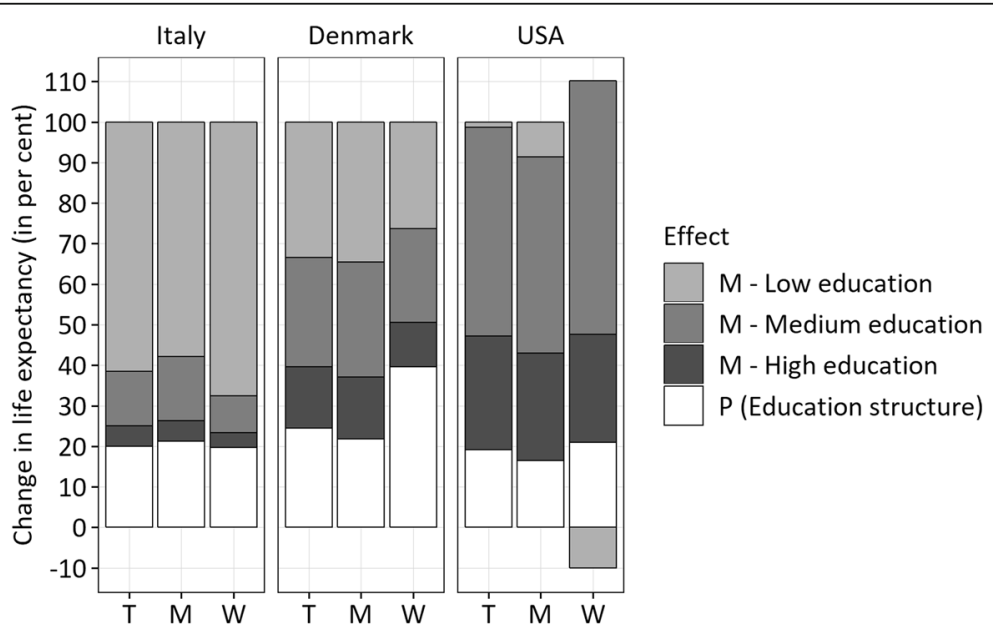

Fig. 2 Relative contributions of changes in mortality ( $M$ effect) and populations' educational structure ( $P$ effect) to differences in life expectancy at age 30 (in percent) for Italy (1991-2011), Denmark (1990-2010), and the USA (1990-2010), stratified by gender. Source: authors' own calculations; the data sources for the three populations can be found in the "Data" section. Note: T refers to total population, $\mathrm{M}$ is men, and W is women 
to the P effect in the USA can be primarily explained by the smaller overall increase in life expectancy (see Table 2). When looking at the relative contributions, the effect of changes in the educational structure on changes in $e_{30}$ was of similar magnitude in all three populations: $19.1 \%$ in the USA, $19.9 \%$ in Italy, and $24.4 \%$ in Denmark.

As shown in Table 3 and Fig. 2, the relative effects are also similar across the male and female populations in all three countries. The only outlier is Danish women where the effect of changing educational composition on increasing life expectancy was particularly high, accounting for almost $40 \%$ of the change in $e_{30}$ between 1990 and 2010. This reflects the fact that of all analyzed populations, Danish women experienced the greatest shifts in educational structure during our study period, with the largest decrease in the proportion of less educated and the largest increase in the proportion of highly educated individuals (see Table 1). Overall, these results illustrate the substantial contributions of progress in educational attainment to the improvements in life expectancy between 1990 and 2010 in all three countries, in addition to all the other forces leading to decreasing overall mortality.

\section{Discussion}

Methodological and conceptual issues

Several technical notes are in order. We performed a series of sensitivity analyses to test whether, and to what extent, our results are affected by the definition used for education levels, the restriction of the decomposition to changes between a start and an end year of a defined observation period, the chosen observation period itself, and the decomposition technique applied. With regard to the latter issue, we tested an alternative approach developed by Das Gupta (1993) which combines the classic age decomposition of a change in life expectancy (Andreev 1982; Pressat 1985) with the general method for decomposing differences between rates (Das Gupta 1978). We compared the results of this method with those derived with the replacement decomposition technique on the basis of the correlation coefficients for the estimated age-specific M and P effects. All coefficients were positive and close to 1.0, the minimum value being 0.9883 for the $\mathrm{M}$ effect of medium-educated women in Denmark. Thus, both methods do not differ with regard to the direction of the estimated effects and they vary only marginally with regard to their magnitudes.

Further, we tested to what extent different definitions of education levels affect the results of our study. We did this with the data for Italy by performing the analysis on the basis of different classifications of education, including a narrower definition of low education (ISCED 0-1) and a broader definition of medium education (ISCED 2-4) as well as the use of four education classes (ISCED 0-1, 2-3, 4, 5-6). In all cases, the estimated $\mathrm{P}$ effects went in the same direction but were larger than in the presented data based on the conventional three education classes (ISCED 0-2, 3-4, 5-6). Note that if a continuous underlying relationship between length of education and level of life expectancy is assumed, then any categorization into discrete education categories will necessarily result in an underestimation of the $\mathrm{P}$ effect. This underestimation gets the stronger the smaller the number of categories is and the broader the education ranges they cover. If over time the average length of education within, e.g., the middle category of education increases, this would also lead to an increase in the life expectancy of the people belonging to this category. Under the three-category-framework, this increase in 
life expectancy would, however, not be classified as an education effect but rather as an exogenous mortality decline effect. If, on the other hand, the middle education category would be split into two, part of this improvement in both education and life expectancy would result in more people moving from the lower middle to the upper middle education category and the resulting life expectancy increase would result in a stronger P effect. The above mentioned results of the sensitivity analysis with four education categories clearly confirm this pattern. More generally, it can be said that the larger the number of education categories-i.e., the more closely it reflects the likely continuous underlying relationship - the larger will be the effect attributed to $\mathrm{P}$.

The data for Italy also allowed us to carry out the decomposition for the 30-year period from 1981 to 2011. This analysis yielded basically identical outcomes in terms of the relative contribution of the increasing education level to rising life expectancy. Finally, a year-by-year decomposition with annual data for Denmark revealed that the $\mathrm{P}$ effect builds up gradually and shows less fluctuations than the $\mathrm{M}$ effect, indicating that our conclusions with regard to the effect of populations' changing educational structure on changing life expectancy are not affected by the direct decomposition of changes between the start and the end year of the study period.

Life tables derived from different data sources for deaths and population at risk as done in the present study for Italy and the USA always entail the risk of a numerator-denominator bias (Sorlie and Johnson 1996). Note that our study focuses on the estimation of the association of increasing education levels with overall life expectancy. Our data are period life tables which model what would happen to a population if a given set of mortality conditions were experienced: the empirical data are an approximate reflection of actual conditions. Therefore, it is most important to estimate for each population education-specific death rates for the start and end years with the same methodological approach, what was only possible in the performed manner. Yet, it seems unlikely that our conclusions are influenced by this issue as well as by possible inaccuracies in the reporting of education levels and our imputation strategy for missing data. Regarding the latter, it is likely that our proportional redistribution of deaths with unknown education level on the basis of the deaths with information about education leads to a slight underestimation of the P effect. ${ }^{9}$ There might be further sources for bias connected to some other technical and conceptual issues (see Jasilionis and Shkolnikov 2016), but we expect these to be of only minor relevance for the studied populations and the quality of the underlying data.

In sum, the sensitivity analyses suggest that none of these methodological and technical issues affected the conclusions of this study, and that the presented magnitude of the $\mathrm{P}$ effect is rather conservative. It is important to note, however, that the estimated life expectancy by education level cannot be compared across the studied populations because of differences in the underlying data characteristics as described in the "Data" section. For the same reason, our levels of education-specific life expectancy can differ from other studies which use different data for the same populations. In fact, our estimates for life expectancy at age 30 by education level provide larger inequalities between the highest and lowest education levels than comparable data from EUROSTAT or OECD (Corsini 2010; Murtin et al. 2017). These deviations are strongest for the populations from Italy and the USA, what indicates that our use of unlinked data leads to an overestimation of differences in life expectancy compared to linked data. 
However, linked data were only available for population subsamples for our earlier observation period. Given that we gave priority to use data that covers the total populations, we could not avoid the use of unlinked data for Italy and the USA. For estimating maximum precise levels of education-specific differences in mortality, such a linkage is preferable to our analysis based on independent data sources for the education-specific risk populations and numbers of death by age (see also Valkonen 2006).

\section{Summary of findings}

This study examined the extent to which changes in the educational structure are related to changes in life expectancy at age 30 in Italy (1991-2011), Denmark (19902010), and the USA (1990-2010). We used the same analytical strategy as Shkolnikov et al. (2006) and Jasilionis et al. (2007) to decompose changes in life expectancy at age 30 into the effects of education-specific mortality changes ( $M$ effect) and the effects of changes in the populations' educational structure ( $\mathrm{P}$ effect). The present study improves upon prior research by (1) testing the association between changes in the educational structure of the populations and changes in life expectancy at adult ages, (2) utilizing data from three different countries which markedly differ in terms of populations' health status and education profile, and (3) including more recent years in the analyses. Our findings contribute to the debate about the role of educational attainment in changing population health status.

Three main findings emerge from our analyses. First, there were considerable changes in the educational composition in all three countries during the study period. The proportion of those with low educational attainment decreased, while the proportion of medium and highly educated individuals increased. There were particularly large increases in Italy and Denmark. Second, life expectancy was distributed in a graded fashion across education groups. Life expectancy was highest for those who had more than high school education, followed by those with medium and low educational attainment levels. Importantly, the gap in $e_{30}$ between the highest and lowest educational levels increased over the two decades of our study period. Third, the results from decomposition analysis show that the structural change in education accounted for a substantial proportion of improvements in overall life expectancy in all three countries. Specifically, we found that populations' changes in educational attainment explained between around 15\% (men in the USA) and approximately 40\% (women in Denmark) of the increases in life expectancy at age 30 between 1990 and 2010.

\section{Interpretation of findings}

The present study provides some extension of our understanding of the mechanisms behind recent improvements in life expectancy. Prior research has tended to focus on medical advancements, in particular developments in the screening, treatment, and prevention of cardiovascular diseases, as major contributors to improved population health (Mackenbach et al. 1988; Vallin and Meslé 2004; Wilmoth 2000). Our results demonstrate the additional importance of education in this process. As described in the fundamental social causes of health inequalities theory, education helps individuals to develop health-related resources, allowing highly educated people to enjoy longer and healthier lives (Link and Phelan 1995). When new health technologies are developed, it 
is likely that people with higher education use resources they own, namely money, knowledge, prestige, power and beneficial social connections, to gain access to life-saving innovations.

The presented findings contain also some policy implications. For instance, they provide support for previous studies which suggested to consider education policy within the framework of health policy (e.g., Lleras-Muney 2005; Schoeni and Ofstedal 2010; Stringhini et al. 2017). The Millennium Development Goals (MDGs) sought to achieve universal primary education, with a special focus on less-developed countries. The post-MDGs international development agenda, Sustainable Development Goals (SDGs), also includes goals related to education, such as ensuring inclusive and equitable quality of education and lifelong learning for all. The present findings suggest that these efforts not only contribute to the expansion of mass education but are likely to lead also to reductions in mortality and an increase in populations' life expectancy.

Building on this conclusion, the findings of our study add also insights into the discussion about the future of human longevity. Two major lines of thought have developed in recent years, which are referred to as "optimistic" and "pessimistic" (see Caselli et al. 2014 for details). In principle, both perspectives agree that mortality will continue to decrease. They differ, however, in their opinions on the time course and magnitude of these improvements. According to the pessimists, future progress will be smaller than in the past because the eradication of infectious diseases in industrialized societies will slow down the potential and the pace of mortality declines (Olshansky et al. 1990). In addition, new risk factors, such as obesity and global pandemic outbreaks, are thought to moderate improvements in human longevity (Olshansky 2005). In contrast, the optimists claim that mortality rates will further decline particularly at older ages, leading to un-damped gains in life expectancy (Christensen et al. 2009; Vaupel 2010). Although debate continues regarding future scenarios of human longevity, the current study provides a so far neglected factor in support of the optimistic perspective. The average years of education in the world are projected to increase from 7.9 years in 2010 to 11.0 years by 2060 (Lutz et al. 2014a). It seems likely that these further increases of the average education level will continue to contribute to the process of mortality reduction (see also Deeg et al. 2013).

Nonetheless, measures and programs to improve the health of less-educated individuals are needed. Despite impressive changes in the distribution of educational attainment during the past 20 years, a large proportion of the studied populations still had less than upper secondary education. Moreover, educational gradients in life expectancy widened during the last decades in many industrialized populations as reflected in the presented estimations (see also Caselli et al. 2014; Shkolnikov et al. 2012). Hendi (2015) and Goldring et al. (2016) have shown that much of these changes in education-specific life expectancy are due to the shifts in the populations' educational structure. Note that, the mortality benefits of education appear to be particularly large for better-educated people, while those with low education continue to be left behind. In this context, it is important to note that the improvements in educational attainment might cause the decreasing proportion of people with low education to become increasingly selected towards adverse health conditions (Deboosere et al. 2009; Hendi 2017).

Some issues should be noted which could provide the ground for fruitful follow-up research. First, our study includes only three countries, though their populations cover 
a broad spectrum of education levels and transitions across modern western nations. Shkolnikov et al. (2006) and Jasilionis et al. (2007) performed a comparable analysis for some northern, central, and eastern European countries between the late 1980s and 1990s. For Finland, they found a similar range of (annual) contributions of the P effect to changes in life expectancy as we did for Italy, Denmark, and the USA. The Czech Republic shows smaller effects of structural changes. For Russia, Estonia, and Lithuania, the $\mathrm{P}$ effects were even larger than in our study but overlaid by the drastic negative $\mathrm{M}$ effects prevalent at that time. Sweden shows the largest effects of education-specific changes in the population structure. Larger effects of the structural changes compared to our estimates were also presented by Klein et al. (2006) for the increase in life expectancy between the female cohorts 1925 and 1955 of Germany, by Turra et al. (2016) for changes in life expectancy among Brazilian women between 1960 and 2010, and by De Grande et al. (2014) for changes between the periods 1991/1996 and 2001/2006 among specific urban and non-urban subpopulations from Belgium. The latter study restricted the analysis, however, to young adults aged 19-34 and decomposed changes in the standardized mortality rates instead of life expectancy. In any case, all estimates available to date refer to industrialized societies. As improvements in educational attainment have also been (and will further be) made in the less-developed countries of the world (Gakidou et al. 2010; Lutz et al. 2014a), including more countries into the analyses-both more-developed and less-developed nations-should be a topic of future investigations.

Second, we partitioned changes in life expectancy only into changes in mortality and educational structure. Although evidence shows that education itself is in part directly related to mortality (Geyer and Peter 1999; Lleras-Muney 2005; van Kippersluis et al. 2011), there is also a strong correlation with other dimensions of socioeconomic status, such as income, occupation, and wealth (Ross and Wu 1995). It would be challenging if it were possible to include these factors into the decomposition. Moreover, the macro-level effect of education on mortality might be moderated through other characteristics of the populations, such as ethnicity or country of birth. For instance, a large literature suggests substantial black-white mortality differences in the USA (Williams 2005), which are associated with mortality differences by education level. Montez et al. (2012) have shown that high school education is a threshold for mortality reduction for blacks in the USA, while whites enjoy mortality benefits from continued education after high school. Such heterogeneity effects are manifold and can arise from different causal pathways.

Finally, we assumed the comparability of education levels across countries during the observation time. Although we used a standardized measurement of education (ISCED-97), educational systems as well as the quality of education may differ significantly. Moreover, the studied populations experienced differences in the timing and dynamics of the process of the educational transition. This point has a notable relevance for the described between-country differences in the education-specific origins of the M effects. Lower secondary education became mandatory in Italy only after the 1950s and until age 16 only after 2007. Therefore, most of the people in the low education group around 1990 had no educational attainment at all, especially among the old and oldest old. By the early 2010s, a large proportion of these cohorts had died and the majority of the less-educated subpopulation had a lower secondary degree. In Denmark 
and the USA, these shifts occurred already earlier. This explains why enrollment into secondary education yielded larger $\mathrm{M}$ effects in Italy than in the other two countries. Thus, our understanding of the education-mortality association on the population level would also be deepened by research that accounts for the context and quality of populations' education and related cohort effects.

To conclude, this study demonstrates that progress in education has made important contributions to increasing life expectancy in Italy, Denmark, and the USA over the past two decades. These findings are in line with the theoretical heterogeneity approach, which states that mortality levels and differences in mortality are strongly influenced by the specific risk group composition of populations (Vaupel et al. 1979; Vaupel and Yashin 1985). In addition to all the other important benefits of education (Lutz 2009; Lutz et al. 2014b), it can also be viewed as a powerful health policy which allows more people to enjoy both better and longer lives. As aptly summarized by Kaplan et al. (2014: 193), "Enhancing health outcomes through improved educational attainment is an attractive alternative, although we still need better evidence that interventions to improve educational attainment will increase life expectancy." Our contribution tried to provide a piece of this evidence.

\section{Endnotes}

${ }^{1}$ Web address: http://dati.istat.it/

${ }^{2}$ Web address: http://www.mortality.org

${ }^{3}$ Web address: https://usa.ipums.org/

${ }^{4}$ Web address: http://www.cdc.gov/nchs/nvss.htm

${ }^{5}$ See: http://www.cdc.gov/nchs/nvss/vital_certificate_revisions.htm

${ }^{6}$ See: https://international.ipums.org/international-action/variables/EDATTAIN\# description_section

${ }^{7}$ We used the Brass model instead of the more commonly used models of Gompertz or Kannisto because of the comparatively young age 65 as starting point for the extrapolation. The Brass model adjusts the survival function of a complete standard life table (in our case life tables for the total populations with single age data from 30 to 110) to fit the available but truncated information about the mortality of the analyzed population (in our case the subpopulations by education level from ages 30 to 64). The Brass model uses two parameters for this adjustment: the alpha parameter reflecting the level of the subpopulations' overall mortality and the beta parameter for the subpopulations' specific age pattern of mortality, both in relation to the standard life table. Luy et al. (2015) have demonstrated the functionality of this approach in their study of life expectancy by education, income, and occupation for the German population. Details about the extrapolation of age-specific probabilities of dying with the Brass model can be found there. Descriptions of the derivation of the Brass parameters alpha and beta from empirical data can be found in Ngom and Bawah (2004) and Stewart (2004).

${ }^{8}$ MS Excel File: Decomposition_replacement_from_young_to_old_ages(4).xls

${ }^{9}$ In an alternative analysis, we assigned all deaths with unknown education to the lowest education level (based on the assumption that the majority of deaths with unknown education level-albeit not all-are likely to belong to this group). This scenario led to huge differences compared to our old model for the population of the USA because the life expectancy of the lowest education decreased strongly for the low 
education group in period 1 (from 41.7 to 34.4 years among men and from 49.5 to 42.9 years among women). Consequently, the low education group experienced by far the strongest increase in life expectancy between period 1 and period 2. This led (1) to an extreme shift of the education-specific $M$ effects from the high to the low education level and (2) regarding the total effects to a shift from the total $\mathrm{M}$ effect to the P effect. Among men of the USA, the P effect increased from 0.6 years in our old model to 2.1 years, and among women of the USA from 0.4 to 1.7 years. For the Italian population, these shifts were considerably smaller (because of the much lower number of deaths with unknown education level in period 1), with the P effects increasing from 1.1 to 1.5 years among men and from 0.7 to 1.2 years among women.

Additional file:

Additional file 1: Basic data and life tables by educational attainment. (CSV $54 \mathrm{~kb}$ )

\title{
Acknowledgements
}

We thank Henrik Brønnum-Hansen (University of Copenhagen) for preparing and providing us the data for Denmark, two anonymous reviewers of this manuscript, and Werner Richter for language editing.

\section{Funding}

This research was supported by the European Research Council, within the European Community's Seventh Framework Programme (FP7/2007-2013), ERC Grant Agreement No. 262663 (HEMOX) and within the EU Framework Programme for Research and Innovation Horizon 2020, ERC Grant Agreement No. 725187 (LETHE).

\section{Availability of data and materials}

All data are available online or in printed form as described in the "Data" section. The life table calculations have been made with MS Excel and the decomposition analyses with the MS Excel macro

"Decomposition_replacement_from_young_to_old_ages(4).xIs" provided by Andreev and Shkolnikov (2012) (see

"Methods" section). All data and results are online available in the Additional file.

\section{Authors' contributions}

$M L$ and GC developed the research idea and designed the study. ML directed and supervised the analyses and wrote large parts of the paper. CW-S performed the analyses, directed a systematic literature review with PubMed, and gave inputs to the paper, in particular the "Methods" section. MZ collected and prepared the data for Italy and the USA, supported the analyses, and gave inputs to the paper. YM reviewed the literature and prepared large parts of the paper. WL and GC contributed to the interpretation of the data and commented on all parts of the paper with corresponding inputs to the text. All authors read and approved the final manuscript.

\section{Competing interests}

The authors declare that they have no competing interests.

\section{Publisher's Note}

Springer Nature remains neutral with regard to jurisdictional claims in published maps and institutional affiliations.

\begin{abstract}
Author details
${ }^{1}$ Wittgenstein Centre for Demography and Global Human Capital (IIASA, VID/ÖAW, WU), Welthandelsplatz 2 / Level 2, 1020 Vienna, Austria. ${ }^{2}$ Vienna Institute of Demography of the Austrian Academy of Sciences, Welthandelsplatz 2 / Level 2, 1020 Vienna, Austria. ${ }^{3}$ International Institute for Applied Systems Analysis, Laxenburg, Austria. ${ }^{4}$ Vienna University of Economics and Business, Welthandelsplatz 2 / Level 3, 1020 Vienna, Austria. ${ }^{5}$ Faculty of Liberal Art, Sophia University, 7-1 Kioicho Chiyodaku, Tokyo 102-8554, Japan. ${ }^{6}$ Department of Statistical Sciences, Sapienza University of Rome, Viale Regina Elena 295, 00161 Rome, Italy. ${ }^{7}$ Department of Methods and Models for Economics, Territory and Finance, Sapienza University of Rome, Via Del Castro Laurenziano 9, 00161 Rome, Italy.
\end{abstract}

Received: 10 October 2018 Accepted: 15 January 2019

Published online: 07 March 2019

\section{References}

Andreev, E. M. (1982). Metod komponent $v$ analize prodoljitelnosty zjizni [the method of components in the analysis of length of life]. Vestnik Statistiki, 9(3), 42-47.

Andreev, E. M., \& Shkolnikov, V. M. (2012). An Excel spreadsheet for the decomposition of a difference between two values of an aggregate demographic measure by stepwise replacement running from young to old ages. MPIDR technical report TR-2012 002. Rostock: Max Planck Institute for Demographic Research. 
Andreev, E. M., Shkolnikov, V. M., \& Begun, A. Z. (2002). Algorithm for decomposition of differences between aggregate demographic measures and its application to life expectancies, healthy life expectancies, parity-progression ratios and total fertility rates. Demographic Research, 7(14), 499-522.

Arendt, J. N. (2005). Does education cause better health? A panel data analysis using school reforms for identification. Economics of Education Review, 24(2), 149-160.

Baker, D. P., Leon, J., Smith Greenaway, E. G., Collins, J., \& Movit, M. (2011). The education effect on population health: a reassessment. Population and Development Review, 37(2), 307-332.

Brass, W. (1971). On the scale of mortality. In W. Brass (Ed.), Biological aspects of demography (pp. 69-110). London: Taylor and Francis.

Caselli, G. (2015). Mortality, epidemiological, and health transitions. In J. D. Wright (Ed.), International encyclopedia of the social \& behavioral Sciences (2nd ed., pp. 857-862). Oxford: Elsevier.

Caselli, G., Drefahl, S., Wegner-Siegmundt, C., \& Luy, M. (2014). Future mortality in low mortality countries. In W. Lutz, W. P. Butz, \& S. KC (Eds.), World population and human capital in the twenty-first century (pp. 226-272). Oxford: Oxford University Press.

Christensen, K., Doblhammer, G., Rau, R., \& Vaupel, J. W. (2009). Ageing populations: the challenges ahead. Lancet, 374(9696), 1196-1208.

Clark, D., \& Royer, H. (2013). The effect of education on adult mortality and health: evidence from Britain. American Economic Review, 103(6), 2087-2120

Corsini, V. (2010). Highly educated men and women likely to live longer. Life expectancy by educational attainment. Statistics in focus 24/2010. Brussels: Eurostat.

Das Gupta, P. (1978). A general method of decomposing a difference between two rates into several components. Demography, 15(1), 99-112.

Das Gupta, P. (1993). Standardisation and decomposition of rates: a user's manual. Current population reports P23-186. Wasghington: U.S. Government Printing Office.

Davey Smith, G., Hart, C., Hole, D., MacKinnon, P., Gillis, C., Watt, G., Blane, D., \& Hawthorne, V. (1998). Education and occupational social class: which is the more important indicator of mortality risk? Journal of Epidemiology and Community Health, 52(3), 153-160.

De Grande, H., Vandenheede, H., \& Deboosere, P. (2014). Trends in young-adult mortality between the 1990s and the 2000s in urban and non-urban areas in Belgium: the role of a changing educational composition in overall mortality decline. Health \& Place, 30, 61-69.

Deboosere, P., Gadeyne, S., \& Van Oyen, H. (2009). The 1991-2004 evolution in life expectancy by educational level in Belgium based on linked census and population register data. European Journal of Population, 25(2), 175-196.

Deeg, D. J. H., van Vliet, M. J. G., Kardaun, J. W. P. F., \& Huisman, M. (2013). Understanding the mortality decline at older ages. Improved life course or improved present period? Annual Review of Gerontology and Geriatrics, 33(1), 261-291.

Denney, J. T., Rogers, R. G., Hummer, R. A., \& Pampel, F. C. (2010). Education inequality in mortality: the age and gender specific mediating effects of cigarette smoking. Social Science Research, 39(4), 662-673.

Gakidou, E., Cowling, K., Lozano, R., \& Murray, C. J. L. (2010). Increased educational attainment and its effect on child mortality in 175 countries between 1970 and 2009: a systematic analysis. The Lancet, 376(9745), 959-974.

Geyer, S., \& Peter, R. (1999). Occupational status and all-cause mortality. A study with health insurance data from NordrheinWestfalen, Germany. European Journal of Public Health, 9(2), 114-118.

Geyer, S., \& Peter, R. (2000). Income, occupational position, qualification and health inequalities—competing risks? (comparing indicators of social status). Journal of Epidemiology and Community Health, 54(4), 299-305.

Goldring, T., Lange, F., \& Richards-Shubik, S. (2016). Testing for changes in the SES-mortality gradient when the distribution of education changes too. Journal of Health Economics, 46, 120-130.

Hayward, M. D., Hummer, R. A., \& Sasson, I. (2015). Trends and group differences in the association between educational attainment and U.S. adult mortality: implications for understanding education's causal influence. Social Science \& Medicine, 127, 8-18.

Hendi, A. S. (2015). Trends in U.S. life expectancy gradients: the role of changing educational composition. International Journal of Epidemiology, 44(3), 946-955.

Hendi, A. S. (2017). Trends in education-specific life expectancy, data quality, and shifting education distributions: a note on recent research. Demography, 54(3), 1203-1213.

House, J. S., Landis, K. R., \& Umberson, D. (1988). Social relationships and health. Science, 241(4865), 540-545.

Human Mortality Database. 2017. University of California, Berkeley (USA), and Max Planck Institute for Demographic Research (Germany). Available at www.mortality.org or www.humanmortality.de (data downloaded on 25 Sept 2017).

Hummer, R. A., \& Lariscy, J. T. (2011). Educational attainment and adult mortality. In R. G. Rogers \& E. M. Crimmins (Eds.), International handbook of adult mortality (pp. 241-261). Dordrecht, Heidelberg, London, New York: Springer.

Janssen, F., \& Kunst, A. E. (2005). Cohort patterns in mortality trends among the elderly in seven European countries, 1950-99. International Journal of Epidemiology, 34(5), 1149-1159.

Jasilionis, D., \& Shkolnikov, V. M. (2016). Longevity and education: a demographic perspective. Gerontology, 62(3), 253-262.

Jasilionis, D., Jdanov, D., \& Leinsalu, M. (2007). Der Zusammenhang von Bildung und Lebenserwartung in Mittel-und Osteuropa. Jahrbuch der Max-Planck-Gesellschaft, 2006-2007, 103-108.

Kaplan, R. M., Spittel, M. L., \& Zeno, T. L. (2014). Educational attainment and life expectancy. Policy Insights From the Behavioral and Brain Sciences, 1(1), 189-194.

Kilpi, F., Silventoinen, K., Konttinen, H., \& Martikainen, P. (2016). Disentangling the relative importance of different socioeconomic resources for myocardial infarction incidence and survival: a longitudinal study of over 300000 Finnish adults. The European Journal of Public Health, 26(2), 260-266.

Kingston, P. W., Hubbard, R., Lapp, B., Schroeder, P., \& Wilson, J. (2003). Why education matters. Sociology of Education, 76(1), 53-70.

van Kippersluis, H., O'Donnell, O., \& van Doorslaer, E. (2011). Long-run returns to education: does schooling lead to an extended old age? Journal of Human Resources, 46(4), 695-721.

Klein, T., Unger, R., \& Schulze, A. (2006). Bildungsexpansion und Lebenserwartung. In A. Hadjar \& R. Becker (Eds.), Die Bildungsexpansion. Erwartete und unerwartete Folgen (pp. 311-331). Wiesbaden: VS Verlag für Sozialwissenschaften. 
Kröger, H., Pakpahan, E., \& Hoffmann, R. (2015). What causes health inequality? A systematic review on the relative importance of social causation and health selection. The European Journal of Public Health, 25(6), 951-960.

Laaksonen, M., Talala, K., Martelin, T., Rahkonen, O., Roos, E., Helakorpi, S., Laatikainen, T., \& Prättälä, R. (2008). Health behaviours as explanations for educational level differences in cardiovascular and all-cause mortality: a follow-up of 60 000 men and women over 23 years. The European Journal of Public Health, 18(1), 38-43.

Lager, A. C. J., \& Torssander, J. (2012). Causal effect of education on mortality in a quasi-experiment on 1.2 million Swedes. Proceedings of the National Academy of Sciences, 109(22), 8461-8466.

Link, B. G., \& Phelan, J. (1995). Social conditions as fundamental causes of disease. Journal of Health and Social Behavior, 35(extra issue), 80-94.

Lleras-Muney, A. (2005). The relationship between education and adult mortality in the United States. Review of Economic Studies, 72(1), 189-221.

Lutz, W. (2009). Sola schola et sanitate: human capital as the root cause and priority for international development? Philosophical Transactions of the Royal Society of London B: Biological Sciences, 364(1532), 3031-3047.

Lutz, W., \& Skirbekk, V. (2014). How education drives demography and knowledge informs projection. In W. Lutz, W. P. Butz, \& S. KC (Eds.), World population and human capital in the twenty-first century (pp. 14-38). Oxford: Oxford University Press.

Lutz, W., Butz, W. P., \& S. KC. (2014a). World population and human capital in the twenty-first century. Oxford: Oxford University Press.

Lutz, W., Muttarak, R., \& Striessnig, E. (2014b). Universal education is key to enhanced climate adaptation. Science, 346(6213), 1061-1062.

Luy, M., Wegner-Siegmundt, C., Wiedemann, A., \& Spijker, J. (2015). Life expectancy by education, income and occupation in Germany: estimations using the longitudinal survival method. Comparative Population Studies, 40(4), 399-436.

Mackenbach, J. P., Looman, C. W. N., Kunst, A. E., Habbema, J. D. F., \& van der Maas, P. J. (1988). Post-1950 mortality trends and medical care: gains in life expectancy due to declines in mortality from conditions amenable to medical intervention in the Netherlands. Social Science \& Medicine, 27(9), 889-894.

Mirowsky, J., \& Ross, C. E. (2003). Education, social status, and health. New York: Aldine de Gruyter.

Montez, J. K., \& Friedman, E. M. (2015). Educational attainment and adult health: contextualizing causality. Social Science \& Medicine Special Issue. Elsevier.

Montez, J. K., Hummer, R. A., \& Hayward, M. D. (2012). Educational attainment and adult mortality in the United States: a systematic analysis of functional form. Demography, 49(1), 315-336.

Murtin, F., Mackenbach, J., Jasilionis, D., \& Mira d'Ercole, M. (2017). Inequalities in longevity by education in OECD countries. Insights from new OECD estimates. OECD statistics working papers 2017/02. Paris: OECD Publishing.

Ngom, P., \& Bawah, A. A. (2004). INDEPTH model life tables for sub-Saharan Africa. Aldershot, Burlington: Ashgate.

OECD. (2016). Education at a glance 2016: OECD indicators. Paris: OECD Publishing.

Olshansky, S. J. (2005). Projecting the future of U.S. health and longevity. Health Affairs, 24(supplement 2), W5R86-W85R89.

Olshansky, S. J., \& Ault, A. B. (1986). The fourth stage of the epidemiologic transition: the age at delayed degenerative diseases. The Milbank Quarterly, 64(3), 355-391.

Olshansky, S. J., Carnes, B. A., \& Cassel, C. (1990). In search of Methuselah: estimating the upper limits to human longevity. Science, 250(4981), 634-640.

Omran, A. R. (1971). The epidemiologic transition. A theory of the epidemiology of population change. The Milbank Memorial Fund Quarterly, 49(4), 509-537.

Omran, A. R. (1983). The epidemiologic transition theory. A preliminary update. Journal of Tropical Pediatrics, 29(6), 305-316.

Pressat, R. (1985). Contribution des écarts de mortalité par âge á la différence des vies moyennes. Population, 4(5), 766-770.

Preston, S. H., Heuveline, P., \& Guillot, M. (2001). Demography: Measuring and modeling population processes. Oxford: Blackwell.

Qian, Z., \& Preston, S. H. (1993). Changes in American marriage, 1972 to 1987: availability and forces of attraction by age and education. American Sociological Review, 58(4), 482-495.

Rogers, R. G., \& Hackenberg, R. (1987). Extending epidemiologic transition theory: a new stage. Social Biology, 34(3-4), 234-243.

Rogers, R. G., Hummer, R. A., \& Nam, C. B. (1999). Living and dying in the USA: behavioral, health, and social differentials of adult mortality. San Diego: Academic Press.

Rogers, R. G., Everett, B. G., Zajacova, A., \& Hummer, R. A. (2010). Educational degrees and adult mortality risk in the United States. Biodemography and Social Biology, 56(1), 80-99.

Ross, C. E., \& Wu, C.I. (1995). The links between education and health. American Sociological Review, 60(5), 719-745.

Ruggles, S., Genadek, K., Goeken, R., Grover, J., \& Sobek, M. (2015). Integrated public use microdata series: Version 6.0 [machinereadable database]. Minneapolis: University of Minnesota.

Ryan, C. L., \& Bauman, K. (2016). Educational attainment in the United States: 2015. Current Population Reports Washington: United States Census Bureau.

Schoeni, R. F. \& Ofstedal, M. B. (2010). Key themes in research on the demography of aging. Demography, 47(supplement), S5-S15.

Shkolnikov, V. M., Andreev, E. M., \& Begun, A. Z. (2003). Gini coefficient as a life table function: computation from discrete data, decomposition of differences and empirical examples. Demographic Research, 8(11), 305-358.

Shkolnikov, V. M., Andreev, E. M., Jasilionis, D., Leinsalu, M., Antonova, O. I., \& McKee, M. (2006). The changing relation between education and life expectancy in central and eastern Europe in the 1990s. Journal of Epidemiology and Community Health, 60(10), 875-881.

Shkolnikov, V. M., Andreev, E. M., Jdanov, D. A., Jasilionis, D., Kravdal, Ø., Vågerö, D., \& Valkonen, T. (2012). Increasing absolute mortality disparities by education in Finland, Norway and Sweden, 1971-2000. Journal of Epidemiology and Community Health, 66(4), 372-378.

Sorlie, P. D., \& Johnson, N. J. (1996). Validity of education information on the death certificate. Epidemiology, 7(4), 437-439.

Stewart, Q. T. (2004). Brass' relational model: a statistical analysis. Mathematical Population Studies, 11(1), 51-72.

Stringhini, S., Carmeli, C., Jokela, M., Avendaño, M., Muennig, P., Guida, F., Ricceri, F., d'Errico, A., Barros, H., Bochud, M., Chadeau-Hyam, M., Clavel-Chapelon, F., Costa, G., Delpierre, C., Fraga, S., Goldberg, M., Giles, G. G., Krogh, V., Kelly-Irving, M., Layte, R., Lasserre, A. M., Marmot, M. G., Preisig, M., Shipley, M. J., Vollenweider, P., Zins, M., Kawachi, I., Steptoe, A., Mackenbach, J. P., Vineis, P., \& Kivimäki, M. (2017). Socioeconomic status and the $25 \times 25$ risk factors as determinants of premature mortality: a multicohort study and meta-analysis of 1.7 million men and women. The Lancet. https://doi.org/ 10.1016/50140-6736(1016)32380-32387. 
Thygesen, L. C., Daasnes, C., Thaulow, I., \& Brønnum-Hansen, H. (2011). Introduction to Danish (nationwide) registers on health and social issues: structure, access, legislation, and archiving. Scandinavian Journal of Public Health, 39(7 suppl), 12-16.

Turra, C. M., Renteria, E., \& Guimarães, R. (2016). The effect of changes in educational composition on adult female mortality in Brazil. Research on Aging, 38(3), 283-298.

UNESCO. (1996). International standard classification of education ISCED 1997. Montreal: UNESCO-UIS.

Valkonen, T. (2006). Social inequalities in mortality. In G. Caselli, J. Vallin, \& G. Wunsch (Eds.), Demography: analysis and synthesis (Vol. 2, pp. 195-206). London: Academic Press.

Vallin, J., \& Meslé, F. (2004). Convergences and divergences in mortality. A new approach to health transition. Demographic Research, 2(2), 11-44.

Vaupel, J. W. (2010). Biodemography of human ageing. Nature, $465(25$ march), 536-542.

Vaupel, J. W., \& Yashin, A. I. (1985). Heterogeneity's ruses: some surprising effects of selection on population dynamics. The American Statistician, 39(3), 176-185.

Vaupel, J. W., Manton, K. G., \& Stallard, E. (1979). The impact of heterogeneity in individual frailty on the dynamics of mortality. Demography, 16(3), 439-454.

Williams, D. R. (2005). The health of U.S. racial and ethnic populations. The Journals of Gerontology Series B: Psychological Sciences and Social Sciences, 60(special issue 2), S53-S62.

Wilmoth, J. R. (2000). Demography of longevity: past, present, and future trends. Experimental Gerontology, 35, 1111-1129.

Woolf, S. H., Johnson, R. E., Phillips, R. L., \& Philipsen, M. (2007). Giving everyone the health of the educated: an examination of whether social change would save more lives than medical advances. American Journal of Public Health, 97(4), 679-683.

Submit your manuscript to a SpringerOpen ${ }^{\circ}$ journal and benefit from:

- Convenient online submission

- Rigorous peer review

- Open access: articles freely available online

High visibility within the field

- Retaining the copyright to your article

Submit your next manuscript at $\boldsymbol{\nabla}$ springeropen.com 